\title{
A photometric study of the light variations of the triple system DG Leo ${ }^{\star} \star \star$
}

\author{
P. Lampens ${ }^{1}$, Y. Frémat ${ }^{1}$, R. Garrido ${ }^{2}$, J. H. Peña ${ }^{3}$, L. Parrao ${ }^{3}$, P. Van Cauteren ${ }^{4}$, J. Cuypers $^{1}$, P. De Cat ${ }^{5,1}$, \\ K. Uytterhoeven ${ }^{5}$, T. Arentoft ${ }^{1,6}$, and M. Hobart ${ }^{7}$ \\ ${ }^{1}$ Koninklijke Sterrenwacht van België, Ringlaan 3, 1180 Brussel, Belgium \\ e-mail: patricia.lampens@oma.be \\ 2 Instituto de Astrofísica de Andalucía, CSIC, Camino Bajo de Huetor, 18008 Granada, Spain \\ 3 Instituto de Astronomía, UNAM Apartado Postal 70-264 México 04510, DF, Mexico \\ 4 Beersel Hills Observatory, Laarheidestraat 166, 1650 Beersel, Belgium \\ 5 Institute of Astronomy, KULeuven Celestijnenlaan 200B, 3001 Leuven, Belgium \\ ${ }^{6}$ Department of Physics and Astronomy, Aarhus University, 8000 Aarhus C., Denmark \\ 7 Facultad de Física, Universidad Veracruzana, Veracruz, Mexico
}

Received 8 November 2004 / Accepted 14 November 2005

\begin{abstract}
Multi-site and multi-year differential photometry of the triple star DG Leo reveals a complex frequency spectrum that can be modelled as the combination of at least three $\delta$ Scuti type frequencies in the range 11.5-13 c/d (with semi-amplitudes of 2-7 mmag) and a superimposed slow variability of larger amplitude. The period of the slow variation fits very well with half the orbital period of the inner spectroscopic binary indicating the presence of ellipsoidal variations caused by the tidally deformed components in a close configuration. These findings, together with the results of a recent spectroscopic analysis (showing that the system consists of a pair of mild Am stars and one A-type component of normal solar composition), infer that DG Leo is an extremely interesting asteroseismic target. Identification of which component(s) of this multiple system is (or are) pulsating and determination of the excited pulsation modes will both contribute to a much better understanding of the non-trivial link between multiplicity, chemical composition, rotation, and pulsation in the lower part of the classical Cepheid instability strip.
\end{abstract}

Key words. stars: binaries: visual - stars: binaries: spectroscopic - stars: variables: $\delta$ Sct - stars: individual: DG Leo technique: photometric

\section{Introduction}

\subsection{Objectives}

A "bonus" of studying stellar pulsations in binary and multiple systems is that one can exploit the dynamical information to obtain independent constraints on the pulsational models via the determination of the physical properties of the components. Multi-year photometry with a good phase coverage of the beat periods of the oscillations, on the one hand, allows accurate frequency analyses of the combined light. A careful spectroscopic analysis will, on the other hand, provide information on the mass and the luminosity ratios, the effective temperature, the chemical composition, and the surface gravity of each component given that the spectrum is composite. If dynamically determined masses of the system can be obtained with sufficiently

* Based on photoelectric observations obtained at San Pedro Martír, Sierra Nevada observatories, and CCD observations at Beersel Hills Observatory, as well as on photoelectric observations with the Belgian MERCATOR telescope.

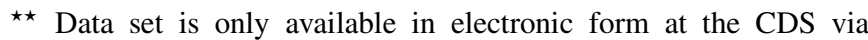
anonymous ftp to cdsarc.u-strasbg.fr $(130.79 .128 .5)$ or via http://cdsweb.u-strasbg.fr/cgi-bin/qcat?]/A+A/438/201 good accuracy, these can be directly confronted with the pulsation masses. By comparing the frequency and modal content of the components that originate from the same protostellar environment, one may realistically hope to improve understanding of pulsation physics, since a difference in pulsational behaviour between each can only be attributed to a limited number of (differing) stellar parameters. Binarity/multiplicity may thus lead to an explanation of the so-far unknown amplitude and mode selection mechanism operating in $\delta$ Scuti stars, and to a refined characterization of the pulsations of variable stars in general.

In a review of pulsating $\delta$ Scuti stars in known double or multiple stars, Lampens \& Boffin (2001) discussed several potentially interesting objects, one of which was DG Leonis which appears to be a triple system whose components are nearly identical.

\subsection{The target}

DG Leo (HR 3889, HIP 48218, Kui $44 \mathrm{AB}$ ) is an hierarchical triple system which consists of a close double-lined spectroscopic binary (components $\mathrm{Aa}$ and $\mathrm{Ab}$ ) and one distant companion (component B) forming the wider visual binary. 
It has $V=6.085 \mathrm{mag}$ and $(B-V)=0.25 \mathrm{mag}$ (Mermilliod \& Mermilliod 1998). The orbital period of the Aa,b system is 4.147 days, while the orbital period of the visual pair $\mathrm{AB}$ is roughly 200 years (Fekel \& Bopp 1977). The visual component $\mathrm{B}$, observed by HIPPARCOS with an angular separation of $0.17^{\prime \prime}$ and a magnitude difference of $0.69 \mathrm{mag}$ with respect to Aa,b (ESA 1997), was monitored by means of micrometric observations and speckle interferometry from 1935 till 1997 (Hartkopf et al. 2000). The existing astrometric data indicate a highly inclined and possibly eccentric orbit. Both Barnes et al. (1977) and Rosvick \& Scarfe (1991) presented evidence for possible shallow eclipses with an inclination of $\approx 75^{\circ}$ for the Aa,b system.

Both close components have spectral type A8 IV (Hoffleit \& Jaschek 1982), while the composite spectrum was classified as type F0 IIIn (Cowley 1976) and type A7 III with enhanced Sr (Cowley \& Bidelman 1979). All three components of the system are located in the $\delta$ Scuti instability strip and are therefore potential candidates for pulsations. Danziger \& Dickens (1967) first reported the short-period variability in the system but were inconclusive about its nature. The claims for short-period oscillations seem to concern mainly one component: component $B$ was classified both as an ultra-short period Cepheid (Eggen 1979 , with a period $<0.275$ days) and as a $\delta$ Scuti star (Elliot 1974 , with a mean periodicity of 0.0818 days). Possible amplitude and phase changes were also reported (Antonello \& Mantegazza 1982; Rosvick \& Scarfe 1991). Since these previous studies are based on very limited photometric data sets, little was known about the pulsational characteristics. A first report on the multiperiodicity of the variations was recently presented after performing a preliminary frequency analysis of newly acquired photometric data (Lampens et al. 2004). In the following sections, we will present the complete and updated photometric analysis of this interesting asteroseismic target, where metallicity as well as binarity effects may influence pulsational behaviour.

\section{Observations and data reduction}

\subsection{Photoelectric photometry}

During 2002 and 2003, we performed photoelectric photometry campaigns of DG Leo at two prime sites located at different longitudes and equipped with identical instrumentation and standard Strömgren filters in a near simultaneous mode. New uvby data were collected with the $1.5 \mathrm{~m}$ telescopes at San Pedro Martír (OAN), México, between Feb. 4-11, 2002 and at Sierra Nevada (OSN), Spain, between Feb. 11-17, 2002. More uvby data were collected at OAN between January 8-14, 2003. We used $\mathrm{C} 1=$ HD $84497=$ HIP $47946=$ GSC 1416-210 (G8 III, $V=7.42, b-y=0.581)$ and $\mathrm{C} 2=\mathrm{HD} 84739=\mathrm{HIP} 48051=$ Cou 284 (F0, $V=7.39, b-y=0.290$, also a close visual double star) as comparison stars. The check star was $\mathrm{C} 3=\mathrm{HD} 86516=$ HIP $48977=$ GSC1418-1085 (Am, $V=6.74, b-y=0.109$ ). The magnitude differences in the sense $(\mathrm{C} 1-\mathrm{C} 3)$ were shown by Rosvick \& Scarfe (1991) to be constant at their level of precision. The HIPPARCOS CATALOGUE (ESA 1997) mentions that $\mathrm{C} 1$ showed no detectable variability throughout the mission,
Table 1. OAN errors in the instrumental system and colour indices of the comparison stars in the standard system (season 2003).

\begin{tabular}{ccccc}
\hline \hline Season & Filter $u$ & Filter $v$ & Filter $b$ & Filter $y$ \\
\hline OAN 2002 & 0.031 & 0.012 & 0.008 & 0.008 \\
OAN 2003 & 0.024 & 0.005 & 0.006 & 0.006 \\
\hline Star & $V\left(\sigma_{V}\right)$ & $(b-y)\left(\sigma_{b-y}\right)$ & $\mathrm{m} 1\left(\sigma_{m 1}\right)$ & $\mathrm{c} 1\left(\sigma_{c 1}\right)$ \\
\hline $\mathrm{C} 1$ & $7.394(20)$ & $0.583(11)$ & $0.326(28)$ & $0.346(45)$ \\
$\mathrm{C} 2$ & $7.358(20)$ & $0.291(11)$ & $0.144(28)$ & $0.473(45)$ \\
$\mathrm{C} 3$ & $6.729(20)$ & $0.097(11)$ & $0.282(28)$ & $0.563(45)$ \\
\hline
\end{tabular}

while the comparison stars C2 and C3 carry the flag "Duplicityinduced variability". The standard deviations of the uvby data of the OSN 2002 and OAN 2003 campaigns show that $\mathrm{C} 1$ and $\mathrm{C} 2$ remained constant at the level of a few mmag, except in filter u for which the noise level is larger (cf. values between brackets in Table 2 introduced later in this section). On the other hand, C3 might be variable on a long-term scale, even though it was used for a short time as a comparison star by Jerzykiewicz (1993). We found a difference of more than $2 \sigma$ on index $\mathrm{m} 1$ and more than $6 \sigma$ on index $\mathrm{c} 1$ when comparing our mean indices with the standard indices published by Maitzen et al. (1998; based on 6 measurements, see below).

The observations at OAN were performed according to the sequence "variable sky C1 variable C2 (variable C3)". Five successive 10-s integrations per filter were used for the stars, while only one 10-s integration per filter was used for the sky. The observations at OSN were performed according to the sequence "variable C1 C2 sky" using 30-s integrations in each filter for all objects. During the OAN 2003 campaign the check star (C3) was also included in the observational sequence.

All OAN data were reduced according to the same reduction method as applied to the OSN data. First, the dead-time correction was applied to all counts. Then, subtraction of the (average) sky measurement from the average value of the target star was carried out for each cycle. The atmospheric extinction was calculated from the comparison star data following the method of Grønbech et al. (1976) and the corresponding corrections were subsequently applied to the magnitude differences. The OAN instrumental errors computed for each season using the standard stars are shown in Table 1. As can be seen from this table, the OAN instrumental errors were larger for the 2002 than for the 2003 season. The reason for the smaller errors in 2003 was the implementation of new phototubes for the uvby photometric system in 2002 followed by a debugging phase which lead to more precise determination of the deadtime corrections for the 2003 OAN season.

The photometric data discussed here represent magnitude differences in the instrumental system between the variable and the comparison star $(\mathrm{C} 1$ or $\mathrm{C} 2)$ interpolated to the observation time of the variable star. As the mean differences were not exactly the same for both observatories, we applied small corrective shifts for each of the three campaigns (see Table 2). 
Table 2. Mean differences between variable/comparison 2 and comparison 1 star per season and per filter.

\begin{tabular}{ccccccc}
\hline \hline Data type & Campaign & No. & $\begin{array}{c}\text { Filter } u \\
\text { Mean (St. dev.) }\end{array}$ & $\begin{array}{c}\text { Filter } v \\
\text { Mean (St. dev.) }\end{array}$ & $\begin{array}{c}\text { Filter } b \\
\text { Mean (St. dev.) }\end{array}$ & $\begin{array}{c}\text { Filter } y \\
\text { Mean (St. dev.) }\end{array}$ \\
\hline V-C1 & OAN 2002 & 429 & $-2.2251(0.022)$ & $-2.2572(0.014)$ & $-1.6962(0.012)$ & $-1.3136(0.011)$ \\
C2-C1 & OAN 2002 & 420 & $-1.1529(0.015)$ & $-0.7820(0.006)$ & $-0.3107(0.005)$ & $-0.0353(0.004)$ \\
\cline { 2 - 7 } V-C1 & OSN 2002 & 748 & $-2.2205(0.011)$ & $-2.2608(0.009)$ & $-1.7454(0.008)$ & $-1.3279(0.007)$ \\
C2-C1 & OSN 2002 & 750 & $-1.0932(0.009)$ & $-0.7351(0.004)$ & $-0.3120(0.003)$ & $-0.0424(0.004)$ \\
V-C1 & OAN 2003 & 269 & $-2.2216(0.016)$ & $-2.2835(0.011)$ & $-1.7666(0.010)$ & $-1.3264(0.008)$ \\
C2-C1 & OAN 2003 & 269 & $-1.1408(0.011)$ & $-0.7843(0.002)$ & $-0.3155(0.002)$ & $-0.0340(0.002)$ \\
\cline { 2 - 7 } V-C1 & OSN 2004 & 144 & $-2.2317(0.010)$ & $-2.2697(0.008)$ & $-1.7509(0.007)$ & $-1.3336(0.006)$ \\
C2-C1 & OSN 2004 & 144 & $-1.1052(0.011)$ & $-0.7407(0.003)$ & $-0.3160(0.004)$ & $-0.0459(0.003)$ \\
\hline
\end{tabular}

Note that the OAN 2003 campaign was held at an epoch closely matching that of the the high-resolution spectroscopic run conducted at the Observatoire de Haute-Provence (Frémat et al. $2004 b$ ). In this way we obtained a homogeneous data set consisting of 1590 epochs and multi-colour differential magnitudes of DG Leo. The new material represents about $110 \mathrm{~h}$ of highquality photoelectric photometry (Data Set 1).

\subsection{CCD photometry}

In order to increase the amount of data and extend the time basis of the previous data set, we also performed complementary observations with a small telescope at Beersel Hills Observatory (BHO), Belgium, during the year 2003. Since the target is very bright, we used a $10 \mathrm{~cm}$ telescope with an SBIG ST10XMe camera to additionally collect differential CCD data, and we adopted C4 = HD $85017=$ HIP $48197=$ GSC1417-315 as the comparison star and C5 = HD $85215=$ HIP 48303 = GSC1417-312 as the check star. We also defocused slightly in order to improve the photometric precision. These frames were reduced with the aperture photometry procedure of the Mira AP software package ${ }^{1} .1071$ differential (stacked) V measurements were thus obtained between Jan. 9 and March 14, 2003 representing a total of $59 \mathrm{~h}$ of differential CCD photometry in the instrumental system. Though the equipment and the filters are not exactly the same and the comparison stars were obviously different, we combined all differential $V$ and Strömgren y measurements into one large set (Data Set 2 containing 2661 differential magnitudes in a "single" filter), which will serve the purposes of a) providing a different time distribution with a distinct alias pattern and b) verifying the frequency solution that will be presented in the following section. To perform such data combination, we needed to apply small corrective shifts of order 0.01 mag on the CCD differential data computed from the shifts between the nightly means of the two comparison stars in the field. The averaged $V$ and Strömgren y magnitude differences were subsequently subtracted for both data types. No other transformation was

1 The software Mira AP is produced by Axiom Research Inc., http: //www . axres.com/ applied. Table 3 shows the log of the observational runs performed between January 2002 and March 2004.

\section{Analyses}

\subsection{Frequency analysis of data set 1}

We performed the frequency analysis with two different codes that are based on computation of the Fourier Tranform and/or on the technique of least-squares fitting.

At first, we used PERIOD98 (Sperl 1998), a programme which determines the frequencies one by one by computing the classical Fourier Transform followed by adjustment of the parameters of the periodic functions (zero point, amplitudes, and phases) using least-squares fitting. Next, the computed frequency solution is refined through simultaneous adjustment of all the parameters, including the frequencies in the course of a (local) minimization process. Since we are dealing with multiperiodic signals, we used the empirical criterion that a frequency is significant as long as the signal-to-noise ratio of the corresponding semi-amplitude is greater than 4 (Kuschnig et al. 1997).

Secondly, a multi-parameter fitting code that scans a wide range in frequency and searches to minimize the residuals by simultaneously adjusting multiple periodic functions was used (global minimization). In this way accurate, alternative multifrequency solutions can be found that might be overlooked by the previous code (Schoenaers \& Cuypers 2004). This is especially useful when an alternative solution due to the ambiguity of some aliasing pattern is also probable. In this case both computations gave identical results in all filters except for filter $u$ which was of lower quality. Table 4 lists the results for Data Set 1 for each filter of the Strömgren system. Listed are the frequency (in $\mathrm{c} \mathrm{d}^{-1}$ ), the semi-amplitude (in mmag), the phase (in $2 \pi \mathrm{rad}$ ), and the total fraction of the variance that is removed from the signal $(\mathrm{R})$. In addition we list the signal-to-noise ratio, with respect to the mean noise level in the residual periodogram computed within a box of width $5 \mathrm{c} \mathrm{d}^{-1}$ centered on the relevant frequency, as well as the standard deviation of the residual signal, $\sigma_{\text {res }}$, which would correspond if PERIOD98 were used; and a succession of prewhitenings with the previous frequency would be performed at each step. 
Table 3. Log of photometric observational campaigns.

\begin{tabular}{cccccccc}
\hline \hline Dates & Observatory & Observer(s) & No. & Comp 1 & Comp 2 & Check & Hrs \\
\hline Feb. 4-11, 2002 & OAN & JP & 429 & C1 & C2 & no & 40.6 \\
Feb. 11-17, 2002 & OSN & RG & 748 & C1 & C2 & no & 42.0 \\
Feb. 22-Mar. 18, 2002 & LPA & PDC and KU & 90 & C1 & no & no & $*$ \\
Jan. 8-14, 2003 & OAN & JP & 269 & C1 & C2 & C3 & 20.0 \\
Jan. 9-Mar. 14, 2003 & BHO & PL and PVC & 1071 & C4 & no & C5 & 58.7 \\
Mar. 17-19, 2004 & OSN & RG & 144 & C1 & C2 & no & 7.0 \\
\hline
\end{tabular}

* Non-continuous observations obtained at La Palma observatory (LPA), cf. Sect. 4.

The dominant frequency F1, the one of largest amplitude found at about $0.48 \mathrm{~cd}^{-1}$, corresponds to a slow periodic change of the mean intensity linked to the orbital variations of the short-period pair $A \mathrm{a}, \mathrm{b}$. It is very close to one half of $P_{\text {orb }}=4.146751$ days, which is the orbital period derived from the published spectroscopic analyses (Fekel \& Bopp 1977; Frémat et al. 2004a). Note that the orbital frequency itself (F0) is not significant, as its signal-to-noise ratio remains far below 4 in all the passbands. However, we considered its contribution, since omitting it would affect the other frequency values very slightly, particularly in the $u$ passband. Four more frequencies between 11.9 and $12.8 \mathrm{c} \mathrm{d}^{-1}$ (F2-F5) were detected in each filter. In fact, two of these are located about $0.08 \mathrm{c} \mathrm{d}^{-1}$ apart, which caused some caution in the preliminary study where $T$ was only about 12 days (Lampens et al. 2004). Their presence is undoubtedly confirmed as the new time base of 767 days corresponds to a formal resolution of $0.0013 \mathrm{~cd}^{-1}$. Also note that F3 and F4 could be subject to the $n$ year $^{-1}$ ambiguity (with $n$ equal to 1 or 3 ). Obviously, there is excellent agreement between the solutions in all four passbands. We point out the high $R$-value in filters $b$ and $v$, but also the poorer match of the same solution in the $u$ passband $(R \sim 33 \%)$. From a comparison of the $R$-values for nearby alias frequency sets, we verified that no other combination was as significant as the one listed for the $y$ passband. As an illustration of this analysis we present Fig. 2, which is entirely based on the computations performed in parallel with PERIOD98 and derived in successive steps for the data in filter $b$.

The expected error in frequency in the case of uncorrelated observations can be computed using the equation

$\sigma_{F}=\frac{\sqrt{6}}{\pi} \cdot \frac{\sigma_{n}}{A \cdot N^{1 / 2} \cdot T}$

(Cuypers 1987), where $A / \sigma_{n}$ indicates the signal-to-noise ratio, $N$, the number of observations and $T$, the total time base. From Table 4 , we adopt $5 \mathrm{mmag}$ as the typical noise level remaining after prewhitening for six frequencies in the filters $v, b$, and $y$ and derive an error in frequency of $1-5 \times 10^{-5} \mathrm{c} \mathrm{d}^{-1}$ on frequencies F1-F5, respectively, given that $T=767$ days and $N=$ 1590 (Data Set 1). This also corresponds to the error one would obtain adopting the formula derived by Schwarzenberg-Czerny (1991), provided that some of the simplifications are not considered. As noted by Schwarzenberg-Czerny (1991) and Montgomery \& O’Donoghue (1999), correlations in the noise

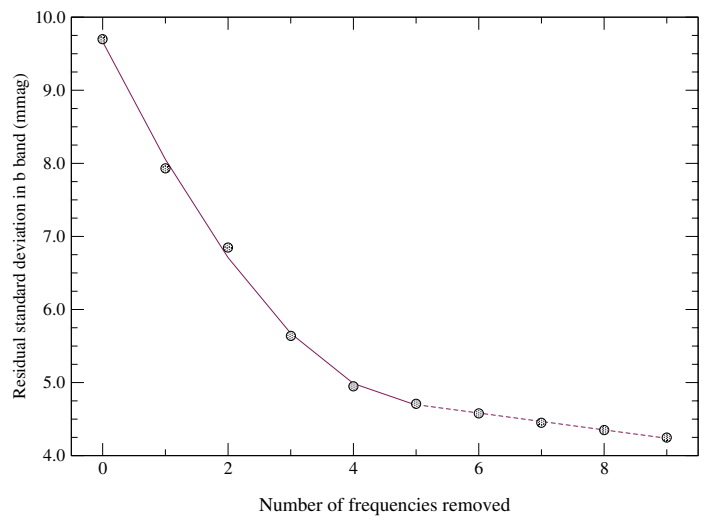

Fig. 1. Residual standard deviation as a function of the number of removed sinusoids (Set 1 - filter $b$ ).

may increase this error with the square root of the noise correlation length $\sqrt{D}$. To estimate $\mathrm{D}$, we analysed the residuals after full prewhitening with six frequencies and found a mean sign change at almost every 4 data points. In the following we will adopt the conservative error estimates of $2-10 \times 10^{-5} \mathrm{~cd}^{-1}$ on frequencies F1-F5. In view of the small differences obtained between the frequency analyses in the filters $y, b$, and $v$, these errors further suggest that a unique solution is valid for all the data. Apart from these values, which can be considered as upper limits of the errors on the frequencies, we recall the fact that the $n$ year $^{-1}$ aliasing effect still affects the current analysis and that this is a practical limitation to the true accuracy of the frequencies F3 and F4 in Table 4. We decided to stop the frequency search at this level since we noted a significant change of the slope at $N=4$ when plotting the residual standard deviation of the $b$ data as a function of the number of sinusoids removed from the original data (Fig. 1).

\subsection{Frequency analysis of data set 2}

Data Set 2 spans the same time base as the former data set but consists of 2661 combined measurements in the filters $y$ and $V$. While the time base is identical, the increased number of measurements should improve the accuracy of the analysis. However, because the site for the CCD data is located near an industrialized area and a smaller telescope was used, the accuracy of a single measurement is lower. We therefore assigned a relative weight of about $5^{-1}$ for each CCD measurement with 
Table 4. Multi-parameter fits and solutions (Data Set 1).

\begin{tabular}{|c|c|c|c|c|c|c|}
\hline $\begin{array}{c}\text { Set } \\
1\end{array}$ & $\begin{array}{l}\text { Freq. } \\
\mathrm{cd}^{-1}\end{array}$ & $\begin{array}{l}\text { Amp. } \\
\text { mmag }\end{array}$ & $\begin{array}{l}\text { Phase } \\
2 \pi \mathrm{rad}\end{array}$ & $S / N$ & $\begin{array}{c}\sigma_{\text {res }} \\
\text { mmag }\end{array}$ & $\begin{array}{l}R \\
\%\end{array}$ \\
\hline & Filter $y$ & \multicolumn{2}{|c|}{$N=1590$} & $\sigma_{\text {init }}=8.4 \mathrm{mmag}$ & & \\
\hline $\mathrm{F} 1$ & 0.482282 & 7.7 & 0.41 & 22 & 6.7 & \\
\hline (F0 & 0.241141 & 0.8 & 0.36 & 2.2 & $-)$ & \\
\hline $\mathrm{F} 2$ & 11.99429 & 5.2 & 0.98 & 13 & 5.8 & \\
\hline $\mathrm{F} 3$ & 11.91064 & 4.5 & 0.63 & 11 & 5.0 & \\
\hline $\mathrm{F} 4$ & 12.11436 & 3.2 & 0.00 & 8 & 4.5 & \\
\hline \multirow[t]{2}{*}{ F5 } & 12.77599 & 1.6 & 0.32 & 4.0 & 4.3 & 74 \\
\hline & Filter $b$ & \multicolumn{2}{|c|}{$N=1590$} & $\sigma_{\text {init }}=9.5 \mathrm{mmag}$ & & \\
\hline $\mathrm{F} 1$ & 0.482276 & 8.4 & 0.41 & 15 & 7.7 & \\
\hline (F0 & 0.241138 & 0.4 & 0.31 & 0.8 & $-)$ & \\
\hline $\mathrm{F} 2$ & 11.99426 & 6.2 & 0.97 & 13 & 6.5 & \\
\hline $\mathrm{F} 3$ & $11.91062^{*}$ & 5.3 & 0.62 & 11 & 5.4 & \\
\hline $\mathrm{F} 4$ & $12.11439^{* *}$ & 3.8 & 0.02 & 8 & 4.7 & \\
\hline \multirow[t]{2}{*}{ F5 } & 12.77592 & 2.3 & 0.34 & 4.9 & 4.4 & 79 \\
\hline & Filter $v$ & \multicolumn{2}{|c|}{$N=1590$} & $\sigma_{\text {init }}=10.8 \mathrm{mmag}$ & & \\
\hline $\mathrm{F} 1$ & 0.482274 & 8.9 & 0.41 & 14 & 9.0 & \\
\hline (F0 & 0.241137 & 0.4 & 0.38 & 0.6 & $-)$ & \\
\hline $\mathrm{F} 2$ & 11.99427 & 7.3 & 0.97 & 13 & 7.6 & \\
\hline F3 & $11.91062^{*}$ & 6.4 & 0.62 & 12 & 6.2 & \\
\hline $\mathrm{F} 4$ & $12.11439^{* *}$ & 4.7 & 0.02 & 9 & 5.3 & \\
\hline \multirow[t]{2}{*}{ F5 } & 12.77591 & 2.6 & 0.34 & 4.8 & 4.9 & 79 \\
\hline & Filter $u$ & \multicolumn{2}{|c|}{$N=1590$} & $\sigma_{\text {init }}=15.5 \mathrm{mmag}$ & & \\
\hline $\mathrm{F} 1$ & 0.482268 & 8.1 & 0.41 & 6 & 14.3 & \\
\hline (F0 & 0.241134 & 2.8 & 0.57 & 2.1 & $-)$ & \\
\hline $\mathrm{F} 2$ & 11.99425 & 6.5 & 0.95 & 7 & 13.7 & \\
\hline $\mathrm{F} 3$ & 11.91055 & 6.3 & 0.59 & 7 & 13.1 & \\
\hline $\mathrm{F} 4$ & 12.11432 & 3.7 & 0.94 & 4 & 12.8 & \\
\hline F5 & 12.77586 & 2.9 & 0.35 & 3.2 & 12.7 & 33 \\
\hline
\end{tabular}

* Alternatively, the 3 (year) $^{-1}$ alias frequency $11.9197 \mathrm{c} \mathrm{d}^{-1}$.

** Alternatively, the 1 (year) ${ }^{-1}$ alias frequency $12.1172 \mathrm{c} \mathrm{d}^{-1}$.

respect to a photoelectric one. The inverse ratio of the variances of the differential magnitudes between the comparison stars $\mathrm{C} 4$ and $\mathrm{C} 5$ (BHO) and $\mathrm{C} 1$ and $\mathrm{C} 2$ (OSN, OAN) was used to compute this factor. The main asset of these data is that the spectral window is different, largely due to the fact that the CCD observations spanned several months in the 2003 season (cf. Fig. 3).

We again performed the same analysis twice: first with PERIOD98, then with the method of multi-parameter fitting. We list the best set of parameters when adopting a six-frequency model with the latter method in Table 5. From a comparison of the $R$-values for close sets of aliased frequencies, we also verified that no other combination was as significant as this one. In particular, the frequency at $12.1145 \mathrm{c} \mathrm{d}^{-1}$ (F4) is slightly more convincing than its year ${ }^{-1}$ alias at $12.1172 \mathrm{c} \mathrm{d}^{-1}$. Note that the 3 year $^{-1}$ ambiguity no longer affects the solution. In theory, the formal errors in frequency would be improved by $\sqrt{N_{1} / N_{2}}$
Table 5. Multi-parameter fit and solution (weighted Data Set 2).

\begin{tabular}{clccccc}
\hline \hline $\begin{array}{c}\text { Set } \\
2\end{array}$ & $\begin{array}{l}\text { Freq. } \\
\mathrm{c} \mathrm{d}^{-1}\end{array}$ & $\begin{array}{c}\text { Amp. } \\
\mathrm{mmag}\end{array}$ & $\begin{array}{c}\text { Phase } \\
2 \pi \mathrm{rad}\end{array}$ & $S / N$ & $\begin{array}{c}\sigma_{\text {res }} \\
\mathrm{mmag}\end{array}$ & $\begin{array}{c}R \\
\%\end{array}$ \\
\hline \multicolumn{7}{c}{ Filter $y+V$} \\
F1 & 0.482278 & 7.6 & 0.42 & 13 & 7.5 & \\
(F0 & 0.241139 & 1.1 & 0.23 & 2.7 & $-)$ & \\
F2 & 11.99425 & 5.0 & 0.98 & 13 & 6.7 & \\
F3 & 11.91064 & 4.0 & 0.63 & 10 & 6.1 & \\
F4 & $12.11446^{*}$ & 2.9 & 0.00 & 8 & 5.8 & \\
F5 & 12.77588 & 1.6 & 0.31 & 4.2 & 5.6 & 62 \\
\hline
\end{tabular}

${ }^{*}$ Alternatively, the 1 (year) $^{-1}$ alias frequency $12.1172 \mathrm{c} \mathrm{d}^{-1}$.

(with $N_{1}$ and $N_{2}$ the number of data in the first and last set respectively). However, considering the use of a relative weight of $5^{-1}$ for the $V$ data, we will adopt the same conservative errors as before (i.e. errors of $2 .-10 \times 10^{-5} \mathrm{c} / \mathrm{d}$ respectively on frequencies F1-F5). From here on, we will adopt the frequency solution of the $v$ data (Data Set 1) as the final solution since it is based on homogeneous data of very high quality and since it is furthermore fully consistent with the solution derived for Data Set 2 within the quoted error bars.

\subsection{Interpretation of the light variability}

As mentioned, the frequency of highest amplitude has a value close to $2 \times f_{\text {orb }}$ and corresponds to a slow periodic variability of the daily mean intensity due to the geometrical variations of the inner binary $A \mathrm{a}, \mathrm{b}$. With an orbital period as short as 4 days, both components of $A \mathrm{a}, \mathrm{b}$ must have their shapes distorted by tidal interaction. We adopted the spectroscopic orbital period $\left(P_{\text {orb }}=4.146751\right.$ days $)$ and used the following model of the light curve of an ellipsoidal binary:

$L_{A \mathrm{a}, \mathrm{b}}(\phi)=\alpha+\beta * \cos (\phi)+\gamma * \cos (2 \phi)$

with $\phi$ the orbital phase and $\alpha$ (the mean light level), $\beta, \gamma$ the coefficients of the fit (Beech 1989). The term corresponding to $2 \times f_{\text {orb }}(\mathrm{F} 1)$ can be associated to the ellipticity effects: the non-spherical cross-sections vary as the system rotates around the centre of mass and maximum luminosity occurs twice per revolution. The term corresponding to $f_{\text {orb }}(\mathrm{F} 0)$ is related to the irradiation effect which occurs once per revolution: the inner part of the cooler component is irradiated by the hotter one and re-emits the excess radiation isotropically. There is no net observable effect in this case, meaning that both components must have similar effective temperatures (Wilson 1994). The question that remains is whether eclipses also occur or not. When fitting the OSN-OAN 2002 data with a single sinusoidal term as a function of half the orbital period, we note the almost constant differences in the nightly means of the OAN run which can be assigned either to shallow eclipses and/or to ellipsoidal variations (Fig. 4), whereas the slow change of the nightly mean levels in the OSN run is evidence for ellipticity effects (Fig. 5). This fit also shows that no (shallow) eclipses are needed to explain the observed light variations, in contrast to a previous 


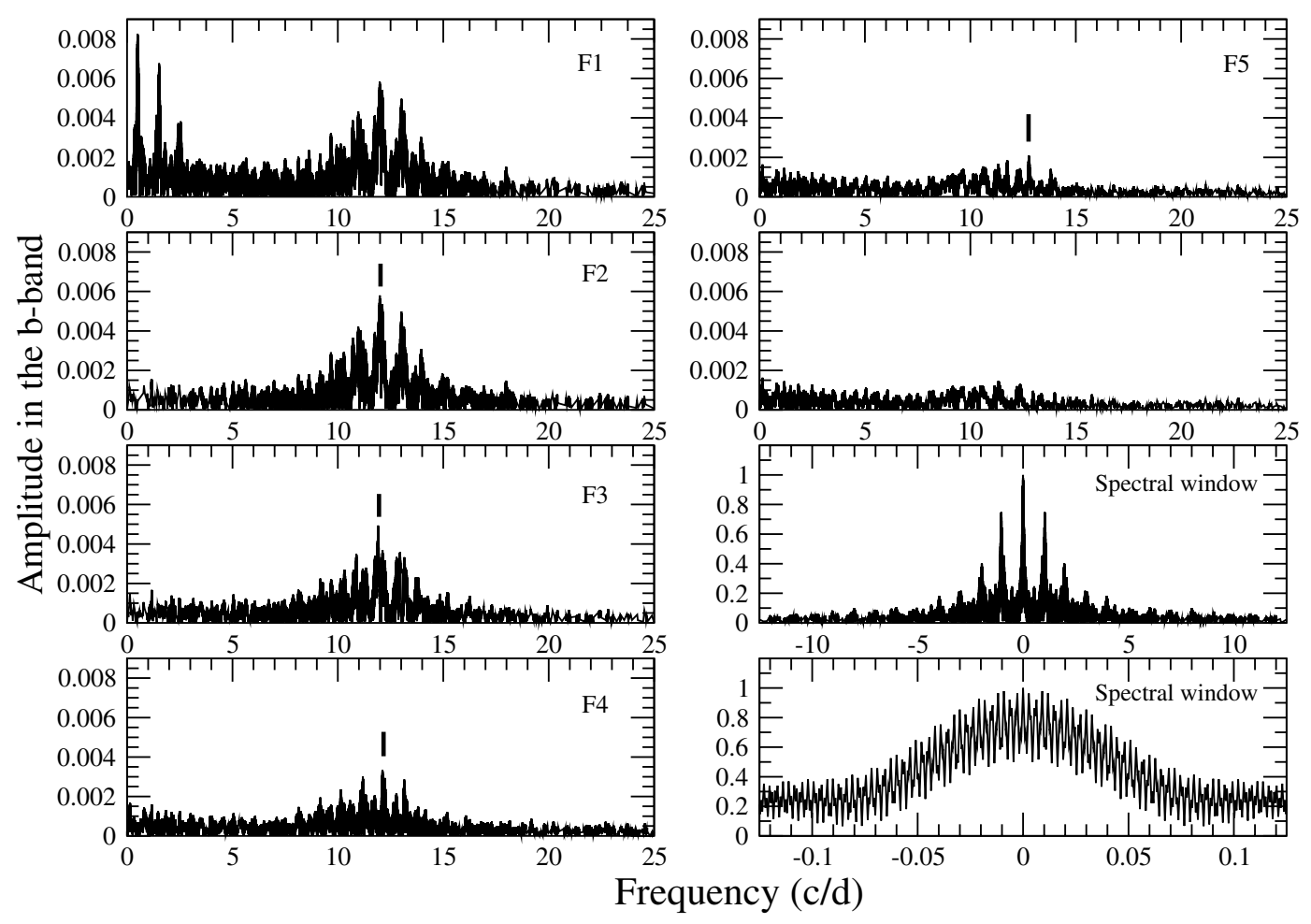

Fig. 2. Successive frequency searches and spectral window of the Fourier analysis (Set $1-$ filter $b$ ).
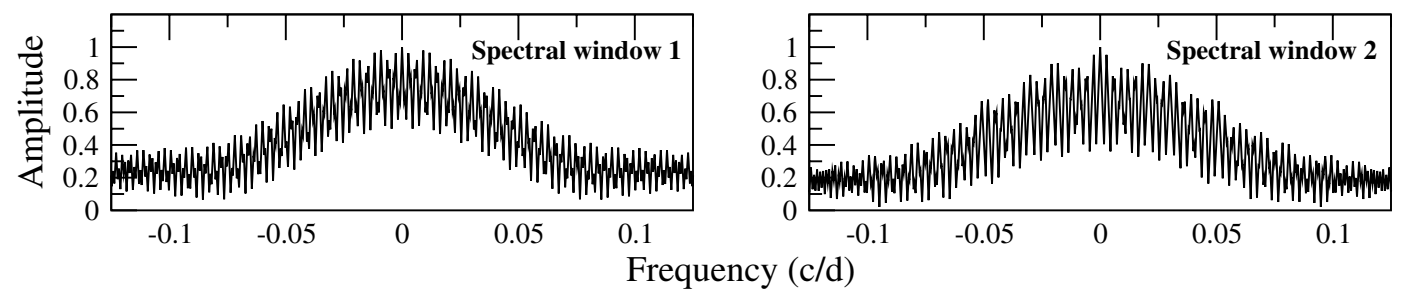

Fig. 3. Spectral windows showing the difference in aliasing between Set 1 and Set 2.

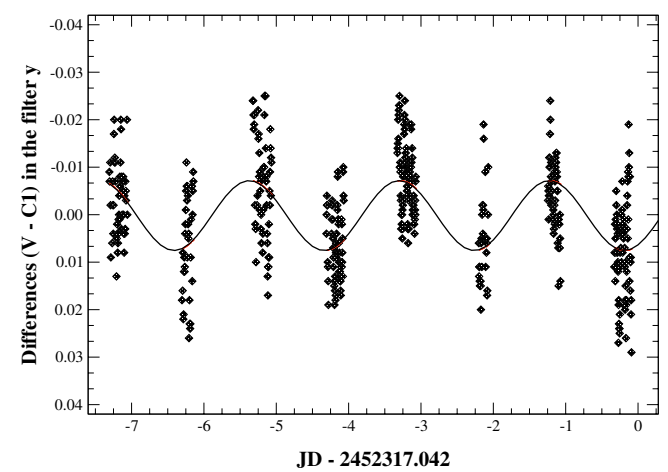

Fig. 4. Individual OAN light curves showing the ellipsoidal variation of DG Leo (filter $y$ ).

estimation (Rosvick \& Scarfe 1991). Thus, the dominant photometric variation can be explained as caused solely by the tidally deformed shapes of both components.

The other frequencies detected in the light variations may be associated to pulsations of the type $\delta$ Scuti in one (possibly more) component(s) of DG Leo. Adopting a unique frequency solution (the final one derived in Sect. 3.2) for all passbands,

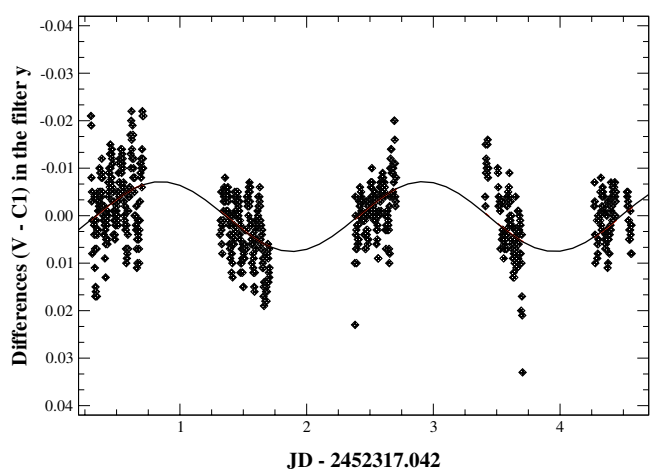

Fig. 5. Individual OSN light curves showing the ellipsoidal variation of DG Leo (filter $y$ ). Note the different orbital phase coverage.

we recomputed the semi-amplitudes and the phases with their standard errors through least squares fitting (Table 6). Note the tiny colour effect in frequencies F1 and F0 as the amplitude in filter $u$ for $\mathrm{F} 0=f_{\text {orb }}$ becomes larger. The amplitude ratios and phase shifts between the different colours displayed in this table contain information that will be helpful for identifying the modes of the excited pulsations (Garrido 2000). Note, 
Table 6. Comparison of amplitudes and phases in various passbands.

\begin{tabular}{ccccccccc}
\hline \hline \multirow{2}{*}{ Set } & \multicolumn{2}{c}{ Filter $u$} & \multicolumn{2}{c}{ Filter $v$} & \multicolumn{2}{c}{ Filter $b$} & \multicolumn{2}{c}{ Filter $y$} \\
1 & $A_{1}$ & $\Phi_{1}$ & $A_{2}$ & $\Phi_{2}$ & $A_{3}$ & $\Phi_{3}$ & $A_{4}$ & $\Phi_{4}$ \\
& $\mathrm{mmag}$ & $2 \pi \mathrm{rad}$ & $\mathrm{mmag}$ & $2 \pi \mathrm{rad}$ & $\mathrm{mmag}$ & $2 \pi \mathrm{rad}$ & $\mathrm{mmag}$ & $2 \pi \mathrm{rad}$ \\
\hline \multirow{2}{*}{$\mathrm{F} 1$} & 8.1 & 0.34 & 8.9 & 0.34 & 8.4 & 0.34 & 7.6 & 0.34 \\
& \pm 0.5 & 0.06 & 0.2 & 0.02 & 0.2 & 0.02 & 0.2 & 0.02 \\
(F0 & 2.8 & 0.43 & 0.4 & 0.62 & 0.4 & 0.69 & 0.8 & $0.64)$ \\
& \pm 0.5 & 0.16 & 0.2 & 0.43 & 0.2 & 0.40 & 0.2 & 0.20 \\
F2 & 6.3 & 0.45 & 7.3 & 0.43 & 6.2 & 0.44 & 5.3 & 0.43 \\
& \pm 0.5 & 0.07 & 0.2 & 0.02 & 0.2 & 0.03 & 0.2 & 0.03 \\
F3 & 6.2 & 0.20 & 6.4 & 0.17 & 5.3 & 0.17 & 4.4 & 0.16 \\
& \pm 0.5 & 0.07 & 0.2 & 0.03 & 0.2 & 0.03 & 0.2 & 0.03 \\
F4 & 3.7 & 0.33 & 4.7 & 0.25 & 3.8 & 0.26 & 3.1 & 0.28 \\
& \pm 0.5 & 0.12 & 0.2 & 0.04 & 0.2 & 0.04 & 0.2 & 0.05 \\
F5 & 2.9 & 0.72 & 2.6 & 0.74 & 2.3 & 0.75 & 1.6 & 0.77 \\
& \pm 0.5 & 0.16 & 0.2 & 0.07 & 0.2 & 0.07 & 0.2 & 0.10 \\
\hline
\end{tabular}

however, that the true semi-amplitudes will be larger than observed since three almost equally luminous stars were observed jointly. Given that $A_{\text {tr }}$ is the true semi-amplitude, $A_{\text {obs }}$ the observed one, and $\mathrm{f}$ the additional light source with respect to the maximum light of the variable component $\mathrm{B}$, we derive:

$A_{\mathrm{tr}}-A_{\mathrm{obs}}=2.5 * \log \frac{1+f \times 10^{0.4 A_{\mathrm{tr}}}}{1+f}$

Application of this equation with $f=2$ gives the corrected semi-amplitudes 0.021 and 0.006 mag corresponding to the extreme values of 0.007 and $0.002 \mathrm{mag}$ for the observed amplitudes of the pulsation, i.e. a factor of 3 larger. On the other hand, the semi-amplitudes related to the ellipsoidal variations should be corrected by an additional 50\% (applying the same equation with $f=0.5$ since the additional light source is component $\mathrm{B}$ with respect to components $A \mathrm{a}, \mathrm{b})$.

\section{Determination of global atmospheric properties}

We next computed the OAN mean magnitudes and colours of the triple star in the Strömgren standard system (for the 2002 season) in order to derive the global atmospheric parameters of the system through application of the calibration procedure (Lester et al. 1986). The absolute magnitude, effective temperature, surface gravity, and metallicity of DG Leo $A \mathrm{ab}, B$ and their standard errors are presented in Table 7.

We also started to collect absolute data in all seven filters of the Geneva photometric system with the MERCATOR telescope $^{2}$. We determined the mean colours and standard deviations of the 90 measurements acquired between February and March 2002 (cf. Table 1). Intrinsic colours of B2 to M0 stars in the Geneva system have been estimated by Meylan et al. (1980) and Hauck (1993). The global colours of DG Leo seem to match an unreddened MK spectral type of A7 III, except

\footnotetext{
2 The MERCATOR telescope is operated by the Institute of
} Astronomy, KU Leuven, Belgium, at La Palma (Spain). for filter $U$ where the system is less luminous (cf. Table 2 in Hauck 1993). We next made use of the CALIB code developed by Künzli et al. (1997) which allows the atmospheric parameters from the Geneva colour indices to be derived for B to mid$\mathrm{G}$ stars on or just above the main sequence. With this code it is also possible to treat metallic-line stars. Using a zero colour excess, we obtained the atmospheric properties listed in Table 7. From the reddening-free parameter $d=1.283$ (measuring the Balmer discontinuity), we next derived $\Delta d=0.107$ (measuring how much the star is evolved away from the main sequence) and $M_{V}=1.51 \pm 0.30 \mathrm{mag}$ following Hauck (1973). However, North et al. (1997) re-examined the existing calibrations of the Strömgren and Geneva systems in terms of $M_{V}$ for Am stars. Their conclusion was that the previous calibrations were not reliable and that the Geneva photometry underestimates the luminosity of the hottest Am stars. Applying the new relation on the Strömgren indices from Table 7 and using $V \sin i \approx 30 \mathrm{~km} \mathrm{~s}^{-1}$ as an appropriate value (Frémat et al. 2004b), we derived the somewhat lower absolute magnitude $M_{V}=1.08 \pm 0.31 \mathrm{mag}$.

We might compare these calibrated mean values with those that can be directly derived on the basis of the Hipparcos parallax (ESA 1997). In Sect. 3.3 we showed that both components of the close binary, $A \mathrm{a}$ and $A \mathrm{~b}$, have similar colours since no net reflection effect is observed in the light curve. We therefore assume that the difference in magnitude between these components is very small (neither one is evolved). On the other hand, we know that the (Hp) magnitude difference between components $A \mathrm{ab}$ and $B$ is $0.69 \mathrm{mag}$ (ESA 1997). Adopting $V=6.085 \mathrm{mag}$, we then obtain $V_{A \mathrm{ab}}=6.546 \mathrm{mag}$ and $V_{B}=$ 7.236 mag. Similarly we also have $V_{A \mathrm{a}} \equiv V_{A \mathrm{~b}}=7.299 \mathrm{mag}$. Converting to absolute visual magnitudes gives $M_{V_{A a}} \equiv M_{V_{A b}}=$ $1.31 \mathrm{mag}$ and $M_{V_{B}}=1.25 \mathrm{mag}$. The relative parallax error of $15 \%$ contributes the most to the error in such a way that none of the above calibrations can be rejected $\left(\sigma\left(M_{V}\right) \approx 2.17 \frac{\sigma_{\pi}}{\pi}=\right.$ $0.33 \mathrm{mag})$.

The listed errors were increased by $50 \%$ with respect to the nominal accuracies to take the fact into account that three stars were observed jointly. Since the three components are not very different, these derived properties will correspond to approximate values of the physical parameters of the components, whereas the high-resolution spectroscopic analysis allowed us to perform a detailed study of the differences between each of the components (cf. Table 6 in Frémat et al. 2004b). A comparison between these component properties and the global values based on photometric calibrations shows that the agreement in effective temperature is reasonably good (mean of $7500 \mathrm{~K}$ ), while the agreement in surface gravity is concordant with the value derived from the Geneva photometry (mean of 3.8). Both $[\mathrm{M} / \mathrm{H}]$ determinations suggest enhanced metallicity of the combined light, an observational fact already mentioned by Fekel \& Bopp (1977) and Cowley \& Bildelman (1979), but only recently quantified (Frémat et al. 2004b).

Acquisition of absolute multi-colour photometry in the Geneva photometric system was pursued during 2004 with the aim of obtaining well-sampled colour curves for each of the pulsation frequencies. The reduction of these observations is presently taking place. 
Table 7. Mean standard colours and global atmospheric parameters of DG Leo in two photometric systems.

\begin{tabular}{|c|c|c|c|c|c|c|c|c|c|c|c|c|}
\hline a) Strömgren & $\begin{array}{c}V \\
\text { mag }\end{array}$ & $\begin{array}{c}b-y \\
\operatorname{mag}\end{array}$ & $\begin{array}{c}m 1 \\
\text { mag }\end{array}$ & $\begin{array}{c}c 1 \\
\operatorname{mag}\end{array}$ & $\begin{array}{c}(b-y)_{0} \\
\text { mag }\end{array}$ & $\begin{array}{c}m_{0} \\
\text { mag }\end{array}$ & $\begin{array}{c}c_{0} \\
\text { mag }\end{array}$ & $\begin{array}{c}\beta \\
\text { mag }\end{array}$ & $\begin{array}{c}M_{V} \\
\text { mag }\end{array}$ & $\begin{array}{c}T_{\text {eff }} \\
\mathrm{K}\end{array}$ & $\begin{array}{c}\log g \\
\operatorname{dex}\end{array}$ & $\begin{array}{c}{[\mathrm{M} / \mathrm{H}]} \\
\operatorname{dex}\end{array}$ \\
\hline & $\begin{array}{l}6.0788 \\
(0.012) \\
\end{array}$ & $\begin{array}{l}0.1462 \\
(0.003) \\
\end{array}$ & $\begin{array}{l}0.2210 \\
(0.005)\end{array}$ & $\begin{array}{l}0.9328 \\
(0.008) \\
\end{array}$ & 0.140 & 0.223 & 0.932 & 2.788 & $\begin{array}{c}1.20 \\
(0.30) \\
\end{array}$ & $\begin{array}{l}7540 \\
(100) \\
\end{array}$ & $\begin{array}{c}3.59 \\
(0.12) \\
\end{array}$ & $\begin{array}{c}0.32 \\
(0.12) \\
\end{array}$ \\
\hline \multirow[t]{2}{*}{ b) Geneva } & $\begin{array}{c}V \\
\text { mag }\end{array}$ & $\begin{array}{c}{[U-B]} \\
\operatorname{mag}\end{array}$ & $\begin{array}{c}{[V-B]} \\
\operatorname{mag}\end{array}$ & $\begin{array}{c}B 1-B \\
\text { mag }\end{array}$ & $\begin{array}{c}B 2-B \\
\text { mag }\end{array}$ & $\begin{array}{c}V 1-B \\
\text { mag }\end{array}$ & $\begin{array}{c}G-B \\
\text { mag }\end{array}$ & & $\begin{array}{l}M_{V} \\
\text { mag }\end{array}$ & $\begin{array}{c}T_{\text {eff }} \\
\mathrm{K}\end{array}$ & $\begin{array}{c}\log g \\
\operatorname{dex}\end{array}$ & $\begin{array}{c}{[\mathrm{M} / \mathrm{H}]} \\
\operatorname{dex}\end{array}$ \\
\hline & $\begin{array}{l}6.0817 \\
(0.012)\end{array}$ & $\begin{array}{l}1.6083 \\
(0.003)\end{array}$ & $\begin{array}{l}0.6467 \\
(0.006)\end{array}$ & $\begin{array}{l}0.9625 \\
(0.002)\end{array}$ & $\begin{array}{l}1.4083 \\
(0.004)\end{array}$ & $\begin{array}{l}1.3656 \\
(0.005)\end{array}$ & $\begin{array}{l}1.7780 \\
(0.006)\end{array}$ & & $\begin{array}{c}1.08 \\
(0.31)\end{array}$ & $\begin{array}{l}7340 \\
(100)\end{array}$ & $\begin{array}{c}3.90 \\
(0.12)\end{array}$ & $\begin{array}{c}0.21 \\
(0.12)\end{array}$ \\
\hline
\end{tabular}

\section{Conclusions and future work}

The multi-colour time series of DG Leo recently collected on two continents at the same epoch were merged together and frequency-analysed. Both time series contain high-quality data in the Strömgren instrumental system. Their combination furthermore presents the advantage of largely suppressed day ${ }^{-1}$ aliasing effects which made the frequency-analyses straightforward and lead (a) to an improved interpretation of (the light curve of) the close binary system and (b) to the detection of multiperiodic oscillations in the combined flux. Monitoring during a few successive years (2002-2004) procured reasonably good accuracy and also permitted verification of the stability of the new frequency solution.

Up to four $\delta$ Scuti frequencies in the range $11.5-13 \mathrm{c} \mathrm{d}^{-1}$ (with semi-amplitudes of 2-7 mmag in the $v$ passband), as well as a slow variation (with a semi-amplitude of $9 \mathrm{mmag}$ in the $v$ passband) were identified with high confidence. The frequency solution as derived from the Strömgren $v$ data appeared to fit the data very well in all other passbands, as well as the more extended data set which is, however, less homogeneous. This unique frequency solution was shown to remain stable over a period of a few years. We further concluded that the period of the slow variation fits very well with half the spectroscopic orbital period of the close binary and that its presence can be explained by continuous ellipsoidal variations supplemented by a tiny colour effect. Despite these efforts, the full frequency content of the pulsations in DG Leo has not yet been identified as still more peaks are present in the same range of the periodogram of the residuals. A far more extended time series would be needed to determine them with good reliability.

As the triple system cannot be resolved photometrically, this analysis is valid for the combined light of all three components. Previous studies indicate that the components have similar characteristics. Therefore, the true semi-amplitudes of the pulsations are diluted by a factor of about 3 . The semiamplitudes and the phases were determined in each of the four Strömgren colour indices and will - after finalising the reduction of the MERCATOR data - also be known in the Geneva photometric system. These will be important diagnostics when identifying the degree $\ell$ of the excited pulsation modes. Of course, such analysis depends on correct identification of the component(s) in which the pulsations originate. Only a detailed spectroscopic analysis can provide reliable and accurate information at the component level. Frémat et al. (2004b) thus revealed that the close binary consists of two mild Am stars and that line-profile variations on the photometric time-scales of the pulsations were detected in the time-series spectra of the distant companion. The in-depth analysis of DG Leo - a triple system with three almost identical components - should put strict empirical constraints on the development of this type of pulsations in stars of the same mass and age, but of different chemical composition in the outer layers.

Our intention is to postpone the modal interpretation until the frequency analysis of the line profile variations detected in the high-resolution spectra can be presented as well. Reliable conclusions on this issue should indeed come from evidence based on both photometric and spectroscopic diagnostics (both types of data were acquired quasi-simultaneously for a better understanding of this triple system). Only then will we be able to consider the exact origin of the pulsations and to establish the link between multiplicity, chemical composition, and pulsation in this extremely interesting $\delta$ Scuti star. A modelling of the pulsations should finally be attempted, in the same way as the preliminary one by Grigahcène et al. (2005).

Acknowledgements. P. Lampens, Y. Frémat, and T. Arentoft gratefully acknowledge financial support from the Belgian Science Policy (through project MO/33/007). R. Garrido aknowledges financial support from the project ESP2001-4528-EP. L. Parrao and J. H. Peña thank the OAN staff for assistance during the observations, Dr. J. P. Sareyan for measuring the OAN dead-time corrections, and PAPIIT IN110102 for the funds provided. Some support from the Fund for Scientific Research - Flanders (Belgium) through project G.0178.02 is also acknowledged. Part of these data was acquired with equipment purchased thanks to a research fund financed by the Belgian National Lottery (1999). Extensive use was made of the ADS and Simbad (operated by the Centre de Données Astronomiques, Strasbourg, France) data bases.

\section{References}

Antonello, E., \& Mantegazza, L. 1982, IBSV, 2152, 1

Barnes, T. G., Fekel, F., \& Moffet, T. J. 1977, PASP, 89, 658

Beech, M. 1989, Astroph. Space Sci., 152, 329

Cowley, A. P. 1976, PASP, 88, 95

Cowley, A. P., \& Bidelman, W. P. 1979, PASP, 91, 83

Cuypers, J. 1987, Medelingen van de Koninklijke Academie voor Wetenschappen, Letteren en Schone Kunsten van België, Jg. 49, Nr. 3 (Brussel: Paleis der Academiën), 21 
Danziger, I. J., \& Dickens, R. J. 1967, IBSV, 150, 1

Eggen, O. J. 1979, ApJS, 41, 413

Elliot, J. E. 1974, AJ, 79, 1082

ESA 1997, The Hipparchos and Tycho Catalogues, SP 1200 (Vol. 1) Fekel, F. C., \& Bopp, B. W. 1977, PASP, 89, 216

Frémat, Y., Lampens, P., Hensberge, H., et al. 2004a, in Variable Stars in the Local Group, ed. D. Kurtz, \& K. Pollard, IAU Coll. 193, ASP Conf. Ser., 310, 395

Frémat, Y., Lampens, P., \& Hensberge, H. 2004b, MNRAS, 356, 545

Garrido, R. 2000, in Delta Scuti and Related Stars, ed. M. Breger, \& M. H. Montgomery, ASP Conf. Ser., 210, 67

Grigahcène, A., Suárez, J. C., Dupret, M.-A., \& Garrido, R. 2005, in Tidal Evolution and Oscillations in Binary Stars, ed. A. Claret, A. Gímenez, \& J.-P. Zahn, ASP Conf. Ser., in press

Grønbech, B., Olsen, E. H., \& Strömgren, B. 1976, A\&AS, 26, 155

Hartkopf, W. I., Mason, B. D., McAlister, H. A., et al. 2000, AJ, 119, 3084

Hauck, B. 1973, in Problems of Calibration of Absolute Magnitudes and Temperature of Stars, ed. B. Hauck, \& B. E. Westerlund, IAU Symp., 54, 117

Hauck, B. 1993, in The MK Process at 50 Years, ed. C. J. Corbally, R. O. Gray, \& R. F. Garrison, ASP Conf. Ser., 60, 157

Hoffleit, D., \& Jaschek, C. 1982, The Bright Star Catalogue, Yale Univ. Obs.

Jerzykiewicz, M. 1993, A\&AS, 97, 421

Künzli, M., North, P., Kurucz, R. L., et al. 1997, A\&AS, 122, 51

Kuschnig, R., Weiss, W. W., Gruber, R., et al. 1997, A\&A, 328, 544
Lampens, P., \& Boffin, H. 2001, in Delta Scuti and Related Stars, ed. M. Breger, \& M. H. Montgomery, ASP Conf. Ser., 210, 309

Lampens, P., Garrido, R., Parrao, L., et al. 2004, in The environment and evolution of double and multiple stars, Rev. Mex. Astron. Astrofís. 21, ed. C. Allen, \& C. Scarfe, IAU Coll., 191, 73

Lampens, P., Frémat, Y., Uytterhoeven, K., et al. 2005, in Tidal Evolution and Oscillations in Binary Stars, ed. A. Claret, A. Gímenez, \& J.-P. Zahn, ASP Conf. Ser., in press

Lester, J. B., Gray, R. O., \& Kurucz, R. L. 1986, ApJS, 61, 509

Maitzen, H. M., Pressberger, R., \& Paunzen, E. 1998, A\&AS, 128, 573

Montgomery, M., \& O’Donoghue, D. 1999, Delta Scuti Newsletter, 13,28

Mermilliod, J. C., \& Mermilliod, M. 1998, The General Catalogue of Photometric Data, new version http://obswww. unige.ch/gcpd/gcpd.html

Meylan, G., Python, M., \& Hauck, B. 1980, A\&A, 90, 83

North, P., Jaschek, C., Hauck, B., et al. 1997, in Hipparcos-Venice '97, ESA SP-402, 242

Schoenaers, C., \& Cuypers, J. 2004, in Variable Stars in the Local Group, ed. D. Kurtz, \& K. Pollard, IAU Coll. 193, ASP Conf. Ser., 310, 283

Sperl, M. 1998, Manual for PERIOD98 http://www.astro.univie.ac.at/ dsn/dsn/PERIOD98

Schwarzenberg-Czerny, A. 1991, MNRAS, 253, 198

Rosvick, J. M., \& Scarfe, C. D. 1991, PASP, 103, 628

Wilson, R. E. 1994, IAPPP Communications, spring 1994 